\title{
Professional Support in Pregnancy Influence Maternal Relation to and Feelings for the Baby after Cesarean Birth: An Intervention Study
}

\section{Stina Thorstensson ${ }^{1,3 *}$, Eva Nissen ${ }^{2}$ and Anette Ekstrom ${ }^{1}$}

${ }^{1}$ School of Life Sciences, University of Skovde, Skovde, Sweden

${ }^{2}$ department of Women's and Children's Health, Division of Reproductive and Perinatal Health Care, Karolinska Institutet, Stockholm, Sweden

${ }^{3}$ School of Health and Medical Sciences, Orebro University, Orebro, Sweden

\begin{abstract}
Background: Cesarean birth may negatively affect mother-infant interactions, while professional support may positively affect these interactions. The aim of this study was to evaluate the effects of a process-oriented training program for antenatal midwives and postnatal nurses on first time mothers' perceptions of professional support and on their relation to and feelings for their baby after a cesarean or a normal birth.
\end{abstract}

Methods: An intervention through process-oriented training for health professionals regarding professional support in childbearing was conducted between 1999 and 2003. Ten municipalities were paired and within each pair, randomly assigned to intervention (five) or control (five) municipality. Mothers having caesarean ( $n=94)$ or normal birth ( $n=301)$ were included. Mothers received routine care (Control Group $=\mathrm{CG}$ ) or care from health professionals having received training (Intervention Group=IG). The mothers answered questionnaires three days, three and nine months after birth. Factor analysis identified three factors: "Taking in baby," "Confidence in relation to baby," and "Feelings for baby."

Results: Mothers in the IG with cesarean birth reported more positive for the "Taking in baby" factor $(p=0.002)$ three days after birth, more positive for the "Confidence in relation to baby" factor $(p=0.004)$ and for the "Feelings for baby" factor $(p=0.004)$ nine months after birth compared to Mothers in the CG. Mothers in the IG reported stronger professional support from health professionals compared to CG.

Conclusion: Our result suggests that improved professional support during pregnancy may buffer negative effects of caesarean birth for first-time mothers in relation to and feelings for the baby.

Keywords: Caesarean birth; Health education; Mother-infant interaction; Parental support; Professional support; Process-oriented training

\section{Introduction}

In Western societies, the incidence of cesarean births increased during the last decades of the $20^{\text {th }}$ century. Cesarean births for first-time mothers in Sweden increased from $6.4 \%$ to $18.8 \%$ from 1974 to 2010 [1]. A cesarean birth is a major surgical procedure with increased risk of morbidity for the mother [2] and might have a negative psychological impact on both the mother [3-6] and her family [4-6]. After a cesarean birth, the mother may initially spend less time with her baby [7], and this may have a negative effect on the contact between them [8]. Furthermore, pain and pain medication could make it difficult for the mother to interact with and breastfeed her baby [9]. A less than optimal hormonal adaptation to breastfeeding has been observed in mothers who have had a cesarean birth [10,11].

The human baby is born with the ability to interact with its mother and a need to receive her response. This interaction is necessary for the baby to form an attachment and for its emotional and biological development [12]; it is important that the mother is able to interact sensitively and adjust to the child [13]. Having a cesarean birth might lead to maternal difficulties in meeting the needs of the baby after birth $[12,14]$. Since the mother is extra-sensitive to the needs of her newborn baby after birth $[15,16]$ a less optimal interaction may affect the establishment of the mother's relationship with her baby $[6,8,17,18]$. To strengthen first time mother's process of becoming a mother [19] as well as her relations ship with her baby, both professional [20] and social support is needed [21].

Social and professional support is an interactive processe that may enhance well-being and create a buffer during stressful life events [21], such as becoming a mother after having had a cesarean birth $[5,8]$. It has been shown that a midwife needs a profound interest in the woman in order to offer professional support [22]. Likewise, the recipient needs to perceive the support as positive in order to benefit from it $[21,23]$, and mothers' perceived helpfulness of received support can be important for their interaction with their babies [24]. In the present study, we have operationalized professional support to include the following two aspects: informational support and emotional support [25].

This study is part of a larger intervention study that includes a process-oriented training program on professional attitudes and support in child bearing for midwives and postnatal nurses from antenatal and child health centres [26-28]. Professionals' personal attitudes affect how they act in patient-care situations [29] and attitudes are based on feelings to and a varying degree of knowledge about a specific phenomenon [30]. Healthcare professionals altered their attitudes towards more facilitating less regulating behaviour, which tended to be stable over time, one year after the training program [26,27]. As a result of the process-oriented training program, the professional support during pregnancy improved mothers perception of professional support after birth with emphasis on preparation for their parental role and the baby's needs, which has a positive effect on mothers relation to and feelings for the baby and the breastfeeding [28,31-33].After a caesarean birth, the mother may be more vulnerable in her relation to and feelings for her baby [8-11]. Therefore, the present study was undertaken to

*Corresponding author: Stina Thorstensson, School of Life Sciences, University of Skovde, Skovde, Sweden and School of Health and Medical Sciences, Orebro University, Orebro, Sweden, E-mail: stina.thorstensson@his.se

Received March 13, 2012; Accepted July 24, 2012; Published August 25, 2012

Citation: Thorstensson S, Nissen E, Ekstrom A (2012) Professional Support in Pregnancy Influence Maternal Relation to and Feelings for the Baby after Cesarean Birth: An Intervention Study. J Nurs Care 1:112. doi:10.4172/2167-1168.1000112

Copyright: (C) 2012 Thorstensson S, et al. This is an open-access article distributed under the terms of the Creative Commons Attribution License, which permits unrestricted use, distribution, and reproduction in any medium, provided the original author and source are credited. 
explore whether improved professional support would have a positive influence on this relationship in the presence of the additional challenge of a cesarean birth, compared to mothers with a normal birth. This would be exploratory for hypothesis-generating purposes. Therefore, this study aimed to evaluate the effects of a process-oriented training program for antenatal midwives and postnatal nurses on first-time mothers' perceptions of professional support and on their relation to and feelings for their baby after a cesarean or a normal birth. The following questions were posed to fulfill the aim;

- How does a process-oriented training for antenatal midwives and postnatal nurses influence first-time mothers' relation to and feelings for the baby in relation to their experience of a cesarean or a normal birth?

- Which factors influence first-time mothers' relation to and feelings for the baby, three days, three and nine months after birth?

\section{Materials and Methodology}

The present study is a sub sample from a larger study. The larger study was carried out as an intervention to compare if health professionals who had gone through a process-oriented training program in professional support in childbearing would change their attitudes in a positive way compared to health professionals who had not gone through this training program [26-28]. Recommendations from the CONSORT group (Consolidated Standards of Reporting Trials) were followed in the study [34]. The present study is focusing on effects of the intervention in regard to first-time mothers' feeling and relation to the baby, on the most vulnerable mothers' i.e. those having had a caesarean birth in comparison of the least vulnerable mothers' i.e. those having had a normal birth.

The study was conducted between 1999 and 2003 in south-western Sweden $[26,27]$. The county where the study took place has 280,000 inhabitants and consists of 13 municipalities. The 10 municipalities included in the study have between 10,000 and 51,000 inhabitants, and their antenatal and child health centers serve both urban/suburban and rural districts. The pregnant women meet with the same midwife at the antenatal center, located outside the hospital, approximately 8 to 11 times during their pregnancy. Midwives and postnatal nurses working at antenatal or child health centers work within one municipality only. Approximately 2,500 births occur annually at the maternity clinic. Most women give birth in the hospital, and the care within the delivery and maternity wards is provided by midwives who the women do not personally know. The maternity clinic offers care to most women giving birth within the hospital area; only women with severe complications or risk of giving birth before 28 gestational weeks are transferred to a regional hospital. Continuous intra-partum labor support by professionals is not offered as a routine in the labor ward of the hospital. The average length of hospital stay after birth is between six hours and four days. After the infant is discharged from the hospital, a postnatal nurse from the child health centers assumes responsibility for the infant's health care and continues to do so until the child is six years of age.

The larger study could be described as having two phases, where the first phase was the randomization of municipalities and completion of the process-oriented training program for antenatal midwives and postnatal nurses, and the second phase was the recruitment of mothers and data collection.
Phase 1: Randomization and the process-oriented training program for the midwives and postnatal nurses in professional support

Based on the findings of a baseline study [35,36], the 10 largest municipalities in the selected area were grouped into pairs according to their size and the duration of breastfeeding in those municipalities. In addition, results from the baseline study did not show any significant differences in the women's perceived support in childbearing and breastfeeding, from the professional in the municipalities involved in the study $[35,36]$.The paired municipalities were randomly designated to intervention (five municipalities) or control (five municipalities) by a person not involved in the study. The randomization was done before any data collection began. Furthermore, antenatal midwives and postnatal nurses were allocated to either intervention or control, depending upon whether their work site had been randomly selected as an intervention municipality or as a control municipality. A process-oriented training program [37] in professional support in childbearing was conducted for the midwives and postnatal nurses from the intervention municipalities from September 1999 through to March 2000. In all, 116 healthcare professionals working in the 10 randomized municipalities were asked to participate in the study (44 midwives and 72 postnatal nurses) and 81 agreed to participate, yielding a $69.8 \%$ participant rate (28 midwives (63.6\%) and 53 (73.6\%) postnatal nurses). During the follow-up of the study, another 26 participants dropped out, resulting in a total response rate of 21/28 midwives and 34/53 nurses. There were no differences of importance in base-line characteristics between those who did not complete the training compared with those who did [27].

The teaching program was composed of evidence-based lectures with collegial discussions on professional stance, reflective processes, problem-solving processes, and practical skills in relation to the provision of support during childbirth and breastfeeding (Appendix 1). This process-oriented training program resulted in more facilitating and less regulating attitudes among participating antenatal midwives and postnatal nurses [26]. There were no clearly detectable changes in society or in the health care organization within childbearing during the data collection period described below which could have influenced the result of the study.

\section{Phase 2: The sample of mothers and the data collection procedures}

The first time mothers included in the study had either been cared for by healthcare professionals at the five intervention municipalities (with the process-oriented training program) as described above or by healthcare professionals at the five control municipalities (without the process-oriented training program). Consequently, the firsttime mothers who were recruited to the study belonged either to the intervention municipalities or to the control municipalities. In the study, all first-time mothers, in intervention group (IG) and control group (CG) met the same professionals at the labor and maternity ward. The difference was that first-time mothers in the IG met specially trained midwives during pregnancy and specially trained postnatal nurses in the year after birth compared to first-time mothers in the CG, who did not meet with specially trained midwives during pregnancy or specially trained postnatal nurses in the year after birth. When the first-time mothers responded to the questionnaires they did not know if their antenatal midwife and postnatal nurse had participated in the process-oriented training program (IG) or not (CG).

Eligible participants for the larger study were Swedish-speaking, first-time mothers who gave birth to a singleton, healthy infant at 
Citation: Thorstensson S, Nissen E, Ekstrom A(2012) Professional Support in Pregnancy Influence Maternal Relation to and Feelings for the Baby after Cesarean Birth: An Intervention Study. J Nurs Care 1:112. doi:10.4172/2167-1168.1000112

Page 3 of 9

term, and had received care from healthcare professionals at either the intervention municipality or the control municipality, as described above. The first-time mothers' names were consecutively retrieved from the hospital register and they were invited to participate in the study from April 2000 through April 2002. Exclusion criteria were first-time mothers who gave birth to children with life-threatening diseases or malformations. The larger study included first-time mothers regardless of mode of delivery. The present subsample study focuses on first time mothers' perception of professional support and first time mothers relation to and feelings for the baby in relation to their experience of a caesarean (cesarean birth: IG $n=33, C G n=61$, in total $n=94$ ), or a normal birth (normal birth IG $\mathrm{n}=116, \mathrm{CG} \mathrm{n}=185$, in total $\mathrm{n}=301$ ). The final sample of the present study totaled 395 first time mothers (Figure 1).

Definitions: normal birth was defined as an uncomplicated vaginal birth at term with a healthy baby (Apgar score $>7$ at 1 minute of age), postpartum bleeding not exceeding $1000 \mathrm{ml}$, no perineal lacerations exceeding laceration of the sphincter ani, and spontaneous birth of the baby with spontaneous discharge of the placenta.

Questionnaires: Three questionnaires were developed for the study. Maternity staff members distributed the first questionnaire to participants on the third day after childbirth. Follow-up questionnaires were sent to the mothers' homes one week before the baby would turn three months and nine months old (Figure 1). One reminder was sent two weeks after the initial questionnaire to the mothers who did not respond to the questionnaire.

\section{Obstetric and demographic data}

Obstetric data were collected from the birth records and demographic background data were collected when the first questionnaire was administered.

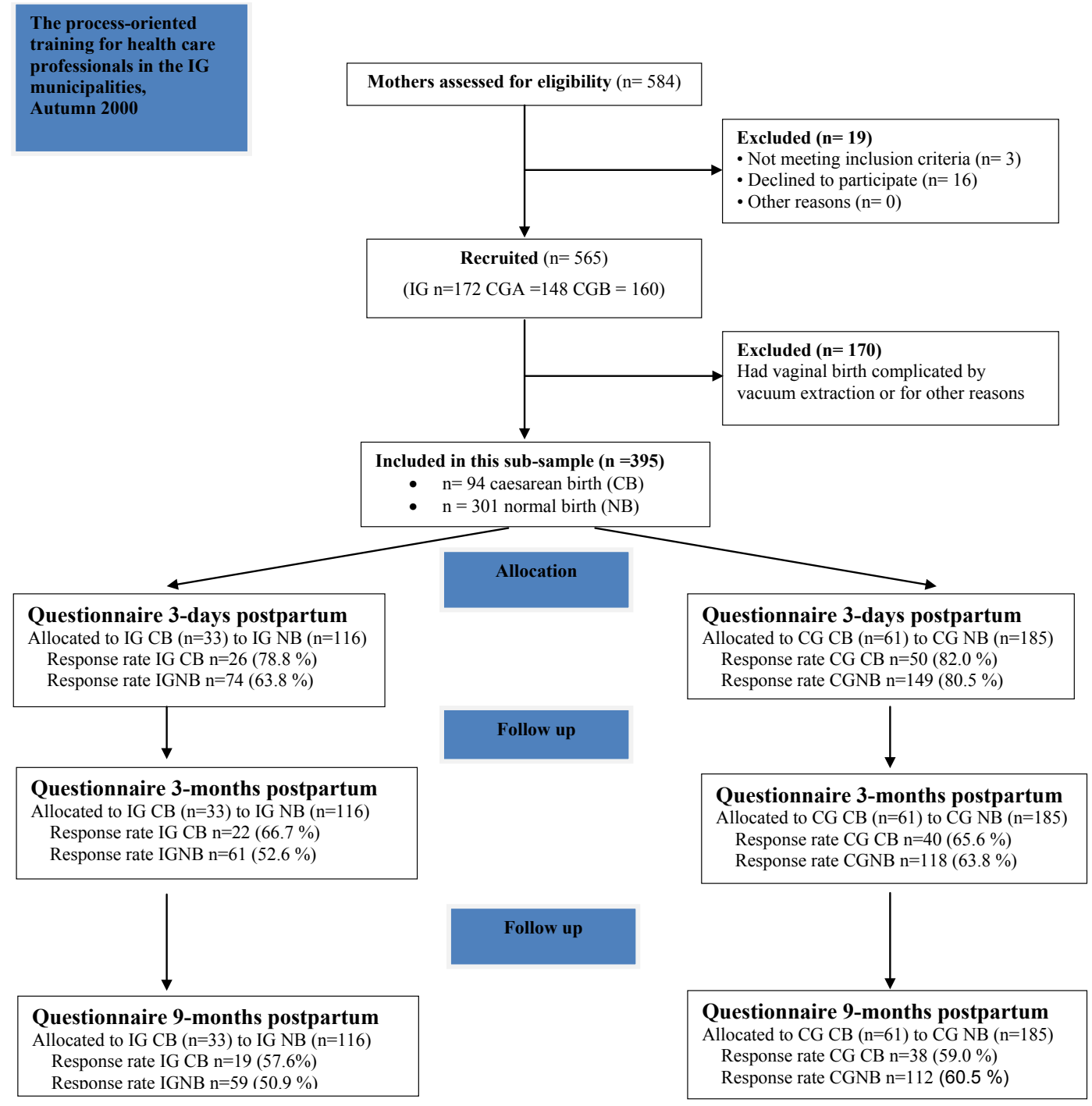

Analysis

Figure 1: Flow figure of response rate of mothers in the control group CG (data collected April 2000 - January 2003 CGA and CGB were merged for this study) and the intervention group (IG) (data collected November 2000 - January 2003) at 3-days, 3-months, and 9-months postpartum. 


\section{Mothers' perceptions of support from the antenatal midwife and the postnatal nurse}

All three questionnaires investigated the mothers' perceptions of support from the antenatal midwife and/or the postnatal nurse. The mothers were asked to reflect on their perception of two aspects of the professional support: emotional support and informational support. Emotional support reflects the individual's emotional experience of receiving care [25]. In this study, the mothers' perceptions of the following caregiver behaviors were assessed: the extent to which the caregivers "were sensitive," "were understanding," "were supportive," "provided time," and "were calm." Informational support refers to the perception of practical advice offered by healthcare professionals [25]. In this study, informational professional support included the mothers' perceptions of "breastfeeding information" and "knowledge of the baby's needs." All items were then rated on a Likert scale ranging from 1 to 7, with 1 signifying "disagree" and 7 signifying "agree completely." For all three sets, the endpoint, "agree completely," sometimes represented a positive assessment and sometimes a negative assessment, thus avoiding the risk of routine-like responses $[38,39]$.

\section{Mothers perceived relationship to and feelings for the baby}

All three questionnaires investigated aspects of the mothers' perception about their relationship with the baby $[15,19,40]$. Participants were asked to respond to statements such as, "I talk a lot with my baby," "I enjoy resting when my baby is with me," "I enjoy breastfeeding," "I feel that my baby is my own," "I know what my baby wants," "My baby is more beautiful than other babies," and "My maternal feelings are very strong." All the items were then rated on a Likert scale ranging from 1 to 7 , with 1 signifying "disagree" and 7 signifying "agree completely." For all three sets, the endpoint, "agree completely," sometimes represented a positive assessment and sometimes a negative assessment, thus avoiding the risk of routine-like responses [38,39]. All three questionnaires investigated aspects of the mothers' perceived feelings for the baby $[15,19,40]$ and asked the participants to respond to the following emotions: "coldwarm," "insecure-secure," "not confident-confident," "difficult-easy," "unstable-stable," and "distant-close." All items were rated on semantic differential scales ranging from 1 to $7[38,39]$.

\begin{tabular}{|l|l|l|l|l|}
\hline Scale Items: Relation to and & \multicolumn{2}{l|}{ Component } & \multirow{2}{*}{ NameUsed in Analysis } \\
\hline Feelings for the Baby & 1 & 2 & 3 & \\
\hline Cold - warm & 0.95 & & & \multirow{2}{*}{ Factor 1 - Taking in baby } \\
\hline I talk a lot with my baby & 0.94 & & & \\
\hline $\begin{array}{l}\text { I enjoy resting when my baby is } \\
\text { with me }\end{array}$ & 0.93 & & & \multirow{2}{*}{ Cronbach's alpha 0.80} \\
\hline I enjoy breastfeeding & 0.92 & & & \\
\hline Insecure - secure & 0.87 & & & \\
\hline I feel that my baby is my own & 0.76 & & & \\
\hline Not confident - confident & & 0.88 & & \multirow{2}{*}{ Factor 2 - Confidence in } \\
relation to baby
\end{tabular}

Extraction method: Principal component analysis; Rotation method: varimax with Kaiser normalization.

Cronbach's alpha was calculated for items within each factor.

Table 1: Results from factor analysis three days after birth.

\section{Statistics}

For the statistical analyses of the results, we used the Statistical Package for the Social Sciences (SPSS, Version 15.0). Chi-square tests were performed on the categorical data. Central measurements were presented as mean (m) and dispersion by Standard Deviation (SD). All items representing the mothers' relation to and feelings for the baby were entered into a principal component factor analysis using varimax rotation in order to reduce the amount of data tested. Three factors with an eigen value $>1$ and a factor-loading $>0.4$ were identified for all 14 items. The tentative naming of the factors is as follows: "Taking in baby" (Factor 1), "Confidence in relation to baby" (Factor 2), and "Feelings for baby" (Factor3). The first factor, "Taking in baby," contained six items. This factor had item loadings ranging from 0.76 to 0.96 . The factor included statements relating to the mothers' relation to and feelings for the baby, such as "I talk a lot with my baby", "I enjoy it when my baby is with me," "cold-warm," "I enjoy breastfeeding," "insecure- secure," and "I feel that my baby is my own". The second factor, "Confidence in relation to baby," contained six items. This factor had item loadings ranging from 0.60 to 0.87 . The factor included statements relating to the mothers' relation to and feelings for the baby, such as "not confident-confident," "difficult-easy," "unstable-stable," "distant-close," "unpleasant-pleasant," and "I know what my baby wants". The third factor, "Feelings for baby," contained two items. This factor had item loadings ranging from 0.65 to 0.83 . The factor included statements relating to the mothers' relation to and feelings for the baby, such as "My baby is more beautiful than other babies" and "My maternal feelings are very strong". To evaluate internal consistency for the factor analysis, Cronbach's alpha was calculated $[38,39]$ and determined to be 0.80 for Factor 1, 0.83 for Factor 2, and 0.61 for Factor 3 (Table 1).

Before comparing the groups, the mean of each subscale was calculated for each individual who participated in the study. Significance between groups was tested using an independent t-test, with twotailed significance. P-values $<0.05$ were considered significant $[38,39]$. Cohen's guidelines were used to calculate the effect sizes in order to interpret clinical change, and the effect was defined as small $(>0.20)$, moderate $(>0.50)$, or strong $(>0.80)[41,42]$.

\section{Pilot test and validation}

The development of the scales was based on recommended practices such as underlying theoretical variables [43], in this study professional support and mothers' feelings and relation to the baby as described above. The three questionnaires were pilot-tested by 20 mothers to determine acceptability and face validity. Minor corrections of the wording of questionnaire items were made before data collection began. To establish content validity, an expert group of midwives, pediatric nurses, pediatricians, and obstetricians was also consulted. The factor analysis performed showed high congruence with the items involved, placing all 14 items within one of the three factors [39]. Ongoing work continues with regard to the validation of the scales, including interviews of mothers [44].

\section{Ethical considerations}

The Ethics Committee of the Medical Faculty of Gothenburg University in Gothenburg, Sweden, approved the study.

\section{Results}

\section{Response rates, demographic and obstetric data}

Response rates for the three questionnaires and the study sample are shown in Figure 1. The demographic and obstetric data for the 
Citation: Thorstensson S, Nissen E, Ekstrom A(2012) Professional Support in Pregnancy Influence Maternal Relation to and Feelings for the Baby after Cesarean Birth: An Intervention Study. J Nurs Care 1:112. doi:10.4172/2167-1168.1000112

Page 5 of 9

participants and the external dropouts did not differ significantly (data not shown). The response rates for the subsamples were $75.7 \%$ for Questionnaire I (three days after birth), 61\% for Questionnaire II (three months after birth), and $57.7 \%$ for Questionnaire III (nine months after birth) (Figure 1). With regard to demographic and obstetrical data, no significant differences existed between the mothers in the IG compared to the mothers in the CG (Table 2).There were no significant differences regarding demographic and obstetrical data, perceived professional support and relation and feelings for the baby, between the mothers who had answered just the first questionnaire compared to those who had answered all three questionnaires.

\section{First-time mothers' perceived professional support}

Caesarean birth: intervention group versus control group: When asked three days after birth, the mothers in the IG with a cesarean birth reported significantly stronger emotional professional support (understanding, $\mathrm{p}=0.005$; time provided, $\mathrm{p}=0.045$; and were calm, $\mathrm{p}=0.004$ ) from the midwife in antenatal care compared to the mothers in the CG. At three months after birth, the mothers in the IG with a cesarean birth reported significantly stronger emotional professional support (were sensitive, $p=0.006$; were supportive, $p=0.011$ ) as well as stronger informational professional support (breastfeeding information, $\mathrm{p}=0.008$ ) from the postnatal nurse compared to the mothers in the CG. At nine months after birth, no significant differences were detected between the IG and the mothers in the CG (Table 3).

Normal birth: intervention group versus control group: When asked three days after birth, mothers in the IG with a normal birth reported stronger emotional professional support ("were supportive," $p$ $=0.008$; "were calm," $\mathrm{p}<0.001$ ) and stronger informational professional support ("breastfeeding information," $\mathrm{p}=0.006$; "knowledge of the baby's needs," $p=0.011$ ) from the antenatal midwife as compared to the mothers in the CG. Three months after birth, mothers in the IG with a normal birth reported stronger emotional professional support ( "were sensitive," $p<0.001$; "were understanding," $p<0.001$ ) as well as informational professional support ("breastfeeding information," $\mathrm{p}=$ 0.001; and "knowledge of the baby's needs, $\mathrm{p}<0.001$ ) from the postnatal nurse as compared to the mothers in the CG. At nine months after birth, mothers in the IG with a normal birth reported significantly more positive for all items used in reference to professional support from the postnatal nurse (Table 3).

Summary: Perceived professional support: mothers' with a caesarean or a normal birth in intervention group versus control group

Three days after birth: mothers in the IG with a caesarean and a normal birth perceived stronger emotional support from the antenatal midwife than mothers in the CG. However, mothers in the IG with a normal birth also perceived stronger informational support than mothers in the CG at this time.

Three months after birth: mothers in the IG with a caesarean and a normal birth perceived stronger emotional as well as informational support from the postnatal nurse than mothers in the CG.

Nine months after birth: mothers in the IG having had a caesarean birth showed no significant differences compared to mothers in the CG. However, mothers in the IG with a normal birth perceived both emotional and informational support stronger than mothers in the CG

\section{First-time mothers' relationship to and feelings for the baby three days after birth}

Caesarean birth intervention group versus control group: At three days after birth, mothers in the IG with a cesarean birth reported significantly more positive for the "Taking in baby" factor as compared to the mothers in the $\mathrm{CG}(\mathrm{p}=0.002)$, with an effect size of 0.57 . At three months, no significant differences were found between the two groups. At nine months after birth, mothers in the IG with a cesarean birth

\begin{tabular}{|c|c|c|c|c|}
\hline Demographicand Obstetric Data & IG CaesareanBirth $\quad(n=33)$ & CG CaesareanBirth $(n=61)$ & IG Normal Birth $(n=116)$ & CG Normal Birth $(n=185)$ \\
\hline Age, in years, mean (SD) & $27.4(4,16)$ & $28.8(4.23)$ & $25.8(4.0)$ & $26.2(5.0)$ \\
\hline Smoked, during pregnancy: yes /no, n (\%) & $2(9.4) / 24(90.6)$ & $2(3.6) / 48(96.4)$ & $4(5.9 / 70(94.1)$ & $3(2.3) / 146(97.7)$ \\
\hline \multicolumn{5}{|l|}{ Education } \\
\hline Compulsory school, n (\%) & $2(7.4)$ & $1(2.1)$ & $4(5.1)$ & $3(2.0)$ \\
\hline High school, n (\%) & $12(48.1)$ & $19(37.5)$ & $35(48.1)$ & $72(48.3)$ \\
\hline University, n (\%) & $9(33.3)$ & $26(52.1)$ & $32(43.0)$ & $55(36.9)$ \\
\hline Other, n (\%) & $3(11.1)$ & $4(8.3)$ & $3(3.8)$ & $19(12.8)$ \\
\hline Total years of education, mean (SD) & $12.7(2.21)$ & $13.31(2.84)$ & $13.0(2.82)$ & $13.0(2.23)$ \\
\hline \multicolumn{5}{|l|}{ Marital status } \\
\hline Cohabiting, n (\%) & 19(75.0) & $32(64.6)$ & $56(75.3)$ & $108(72.7)$ \\
\hline Married, n (\%) & $6(21.4)$ & $17(33.3)$ & $16(22.1)$ & $36(24.0)$ \\
\hline Single, $\mathrm{n}(\%)$ & $1(3.6)$ & $1(2.1)$ & $1(1.3)$ & $2(1.3)$ \\
\hline Other, n (\%) & 0 & 0 & $1(1.3)$ & $3(2.0)$ \\
\hline \multicolumn{5}{|l|}{ Obstetric data } \\
\hline Gestational weeks, mean (SD) & $40.6(1.6)$ & $40.5(1.57)$ & $40.2(1.36)$ & $40.3(1.33)$ \\
\hline Hours of active labour, mean (SD) & $10.5(8.16)$ & $14.0(8.57)$ & $8.19(4.91)$ & 7.99(3.95) \\
\hline Caesarean birth planned, n (\%) & $3(9.1)$ & $15(24.6)$ & & \\
\hline Caesarean birth unplanned, n (\%) & $30(90.9)$ & $46(75.4)$ & & \\
\hline Apgar Score $<7$ at 1 min of age, $n$ & 5 & 8 & 0 & 0 \\
\hline Apgar Score $<7$ at 5 min of age, $n$ & 2 & 2 & 0 & 0 \\
\hline Apgar Score $<7$ at 10 min of age, $n$ & 0 & 0 & 0 & 0 \\
\hline Skin-to-skin after birth yes, n (\%) & $3(12.5)$ & $8(16.3)$ & 73(94.8) & $145(98.6)$ \\
\hline Skin-to-skin after birth no, n (\%) & $22(87.5)$ & $41(83.7)$ & & $2(1.4)$ \\
\hline
\end{tabular}

P-value by independent t-test or Pearson chi-square test. There were no significant differences at $95 \%$ confidence interval between the IG and the CG in demographic and obstetric data.

Table 2: Demographic and obstetric data for intervention group (IG) and control group (CG). 
Citation: Thorstensson S, Nissen E, Ekstrom A(2012) Professional Support in Pregnancy Influence Maternal Relation to and Feelings for the Baby after Cesarean Birth: An Intervention Study. J Nurs Care 1:112. doi:10.4172/2167-1168.1000112

Page 6 of 9

\begin{tabular}{|c|c|c|c|c|c|c|c|c|c|c|}
\hline \multirow{3}{*}{\begin{tabular}{|l|}
$\begin{array}{l}\text { Questionnaires/ Professional } \\
\text { Support }\end{array}$ \\
Three days after birth \\
\end{tabular}} & \multicolumn{5}{|c|}{ Caesarean Birth } & \multicolumn{5}{|c|}{ Normal Birth } \\
\hline & \multicolumn{2}{|c|}{$I G=27$} & \multicolumn{2}{|c|}{$C G=48$} & \multirow[b]{2}{*}{ p } & \multicolumn{2}{|c|}{ IG = 74} & \multicolumn{2}{|c|}{$I G=149$} & \multirow[b]{2}{*}{$p$} \\
\hline & m & sd & m & sd & & m & sd & m & sd & \\
\hline Were sensitive & 5.96 & 1.09 & 5.40 & 1.51 & 0.065 & 5.83 & 1.18 & 5.95 & 1.13 & 0.472 \\
\hline Were understanding & 6.22 & 0.80 & 5.46 & 1.46 & $0.005^{*}$ & 6.00 & 1.06 & 6.09 & 1.11 & 0.539 \\
\hline Were supportive & 5.52 & 1.97 & 4.62 & 1.98 & 0.065 & 5.50 & 1.69 & 4.79 & 2.14 & $0.008^{*}$ \\
\hline Time provided & 6.11 & 1.42 & 5.37 & 1.54 & $0.045^{*}$ & 5.76 & 1.31 & 5.54 & 1.43 & 0.252 \\
\hline Were calm & 6.15 & 1.56 & 4.83 & 2.17 & $0.004^{*}$ & 6.10 & 1.51 & 4.98 & 2.21 & $<0.001^{*}$ \\
\hline Breast feeding information & 5.00 & 1.98 & 4.34 & 2.08 & 0.185 & 5.00 & 1.85 & 4.23 & 1.99 & $0.006^{*}$ \\
\hline \multirow[t]{2}{*}{ Knowledge of the babies needs } & 4.77 & 1.85 & 4.31 & 1.47 & 0.236 & 5.17 & 1.42 & 4.62 & 1.56 & $0.011^{*}$ \\
\hline & \multicolumn{2}{|c|}{ IG = 22} & \multicolumn{2}{|c|}{ CG $=39$} & & \multicolumn{2}{|c|}{$I G=61$} & \multicolumn{2}{|c|}{ CG = 118} & \\
\hline Three months after birth & m & sd & m & sd & $\mathbf{p}$ & m & sd & m & sd & $p$ \\
\hline Were sensitive & 6.45 & 0.80 & 5.59 & 1.27 & $0.006^{*}$ & 6.10 & 0.98 & 5.41 & 1.57 & $<0.001^{*}$ \\
\hline Were understanding & 6.27 & 0.88 & 5.69 & 1.22 & 0.055 & 6.21 & 0.93 & 5.54 & 1.44 & $<0.001^{*}$ \\
\hline Were supportive & 6.29 & 1.19 & 5.31 & 1.66 & $0.011^{*}$ & 5.54 & 1.75 & 5.22 & 1.76 & 0.248 \\
\hline Time provided & 5.86 & 1.70 & 5.45 & 1.37 & 0.304 & 5.73 & 1.50 & 5.29 & 1.69 & 0.087 \\
\hline Were calm & 6.27 & 0.98 & 6.20 & 1.05 & 0.807 & 6.20 & 1.38 & 5.80 & 1.56 & 0.093 \\
\hline Breast feeding information & 6.14 & 1.53 & 4.87 & 1.93 & $0.008^{*}$ & 5.66 & 1.67 & 4.71 & 1.77 & $0.001^{*}$ \\
\hline \multirow[t]{2}{*}{ Knowledge of the babies needs } & 6.00 & 1.27 & 5.49 & 1.21 & 0.124 & 6.22 & 1.12 & 5.37 & 1.52 & $<0.001^{*}$ \\
\hline & \multicolumn{2}{|c|}{$I G=19$} & \multicolumn{2}{|c|}{$C G=36$} & & \multicolumn{2}{|c|}{$I G=60$} & \multicolumn{2}{|c|}{$C G=112$} & \\
\hline Nine months after birth & m & sd & m & sd & $\mathbf{p}$ & m & sd & m & sd & $p$ \\
\hline Were sensitive & 6.05 & 0.91 & 5.44 & 1.48 & 0.069 & 6.03 & 1.09 & 5.25 & 1.45 & $<0.001^{*}$ \\
\hline Were understanding & 6.16 & 0.90 & 5.81 & 1.17 & 0.256 & 6.13 & 0.91 & 5.42 & 1.38 & $<0.001^{*}$ \\
\hline Were supportive & 6.00 & 1.29 & 5.39 & 1.46 & 0.131 & 5.72 & 1.62 & 4.96 & 1.63 & $0.004^{*}$ \\
\hline Time provided & 5.68 & 0.94 & 5.31 & 1.41 & 0.242 & 5.72 & 1.21 & 5.26 & 1.44 & $0.038^{*}$ \\
\hline Were calm & 5.89 & 1.29 & 5.64 & 1.55 & 0.541 & 6.18 & 1.24 & 5.67 & 1.30 & $0.014^{*}$ \\
\hline Breastfeeding information & 5.58 & 1.54 & 4.94 & 1.51 & 0.149 & 5.55 & 1.66 & 4.89 & 1.48 & $0.009^{*}$ \\
\hline Knowledge of the babies needs & 5.84 & 1.42 & 5.55 & 1.38 & 0.473 & 5.88 & 1.37 & 5.22 & 1.42 & $0.004^{*}$ \\
\hline
\end{tabular}

Significance tested with independent t-test. Significance marked with an asterisk $\left({ }^{*}\right)$

Table 3: Mothers' (Caesarean or normal birth) perceptions of the professional support received from the antenatal midwife (retrospective at three days) and the postnatal nurse (at three and nine months after birth) for the intervention group versus the control group.

\begin{tabular}{|c|c|c|c|c|c|c|c|c|c|c|}
\hline \multirow{3}{*}{$\begin{array}{l}\text { Factors } \\
\text { Three days after birth }\end{array}$} & \multicolumn{5}{|c|}{ Caesarean birth } & \multicolumn{5}{|c|}{ Normal birth } \\
\hline & \multicolumn{2}{|c|}{ IG = 26} & \multicolumn{2}{|c|}{$C G=48$} & & \multicolumn{2}{|c|}{$I G=74$} & \multicolumn{2}{|c|}{$C G=149$} & \multirow[b]{2}{*}{$p$} \\
\hline & m & sd & m & sd & $\mathrm{p}$ & m & sd & m & sd & \\
\hline Taking in baby & 5.88 & 1.07 & 4.78 & 1.94 & $0.002^{*}$ & 6.24 & 0.97 & 4.97 & 2.20 & $<0.001^{*}$ \\
\hline Confidence in relation to baby & 5.93 & 0.66 & 5.91 & 0.82 & 0.895 & 6.40 & 0.52 & 6.18 & 0.76 & $0.013^{*}$ \\
\hline Feelings for baby & 6.36 & 1.32 & 6.42 & 0.74 & 0.796 & 6.56 & 0.78 & 6.56 & 0.67 & 0.997 \\
\hline Three months after birth & \multicolumn{2}{|c|}{ IG = 22} & \multicolumn{2}{|c|}{ CG $=39$} & & \multicolumn{2}{|c|}{ IG = 61} & \multicolumn{2}{|c|}{$C G=118$} & \\
\hline Taking in baby & 6.23 & 0.63 & 6.33 & 0.63 & 0.556 & 6.55 & 0.41 & 6.34 & 0.65 & $0.009^{*}$ \\
\hline Confidence in relation to baby & 6.53 & 0.67 & 6.45 & 0.53 & 0.601 & 6.69 & 0.33 & 6.58 & 0.54 & 0.099 \\
\hline Feelings for baby & 6.57 & 0.89 & 6.69 & 0.56 & 0.529 & 6.70 & 0.68 & 6.65 & 0.61 & 0.662 \\
\hline Nine months after birth & \multicolumn{2}{|c|}{ IG $=19$} & \multicolumn{2}{|c|}{ CG $=37$} & & \multicolumn{2}{|c|}{ IG = 59} & \multicolumn{2}{|c|}{$C G=112$} & \\
\hline Taking in baby & 6.41 & 0.66 & 6.32 & 0.73 & 0.675 & 6.49 & 0.45 & 6.26 & 0.64 & $0.007^{*}$ \\
\hline Confidence in relation to baby & 6.85 & 0.17 & 6.52 & 0.63 & $0.004^{*}$ & 6.75 & 0.37 & 6.63 & 0.49 & 0.093 \\
\hline Feelings for baby & 6.84 & 0.33 & 6.40 & 0.76 & $0.004^{*}$ & 6.61 & 0.90 & 6.58 & 0.75 & 0.796 \\
\hline
\end{tabular}

Significance tested with independent t-test. Significance marked with an asterisk $\left({ }^{*}\right)$

Table 4: The factors "Taking in baby", "Confidence in relation to baby" and "Feelings for baby" for the intervention group (IG) versus the control group (CG) in relation to Caesarean or Normal birth, three days, three and nine months after birth.

reported significantly more positive for the "Confidence in relation to baby" factor $(\mathrm{p}=0.004)$ and for the "Feelings for baby" factor $(\mathrm{p}=0.004)$ as compared to mothers in the CG, with an effect size of 0.52 and 0.58 , respectively (Table 4 ).

Normal birth intervention group versus control group: At three days after birth, mothers in the IG with a normal birth reported significantly more positive for the "Taking in baby" factor $(\mathrm{p}<0.001)$ and for the "Confidence in relation to the baby" factor $(\mathrm{p}=0.013)$, as compared to mothers in the CG, with an effect size of 0.59 and 0.29 , respectively. At three months after birth, mothers in the IG with a normal birth reported significantly more positive for the "Taking in baby" factor ( $p=0.009$ ), as compared to mothers in the CG, with an effect size of 0.32 . At nine months after birth, mothers in the IG with a normal birth reported significantly more positive for the "Taking in baby" factor ( $\mathrm{p}=0.007$ ), as compared to mothers in the CG, with an effect size of 0.36 (Table 4).

Summary: Reported relation to and feelings for the baby: mothers' with caesarean or a normal birth in intervention groups versus control groups:

Three days after birth: mothers in the IG with a caesarean or a normal 
birth reported stronger for the "Taking in baby" factor compared to mothers in the CG. Mothers in the IG with a normal birth also reported stronger for the "Confidence in relation to baby" factor compared to mothers in the CG.

Three months after birth: mothers in the IG with a normal birth reported stronger for the "Taking in baby" compared to the mothers in the CG.

Nine months after birth: mothers in the IG with a caesarean birth reported stronger for the "Confidence in relation to baby" and "Feelings for baby" compared to mothers in the CG. Mothers in the IG with a normal birth reported stronger for the "Taking in baby" factor compared to mothers in the CG.

\section{Discussion}

A main result of this study was that mothers in the IG perceived stronger professional support which seemed to strengthen the mothers' relation to and feelings for the baby after birth despite the additional challenge of having had a caesarean birth. IG mothers with caesarean birth reported higher for the "taking in baby factor" than CG mothers with a caesarean birth did, which suggests that the intervention could to buffer the negative impact caesarean birth could have in relation to interaction with the baby. At nine months after birth mothers with a caesarean birth in the IG reported stronger for the "Confidence in relation to baby" and stronger for "Feelings for baby" factors than mothers in CG. Mothers with a caesarean birth showed less prominent differences in the variables assessed than mothers with a normal birth.

The result of this study showed that mothers in the IG with a caesarean or a normal birth perceived a stronger professional support from the antenatal midwife and the postnatal nurses than the mothers in the CG group. These results may depend on the healthcare professionals' personal attitudes which affect how they act in patientcare situations [29]. In order to improve professional support, an approach that addresses the attitude of the professionals is needed [45]. Sometimes, it is difficult for the provider of the support to detect and understand the recipient's support needs $[46,47]$. When caregivers offer support in care situations generally applicable to their own private life, it is important for them to reflect on their personal experience $[12,26]$. Doing so increased the professionals' capacity to provide the support which mothers requested [26]. Altering attitudes can be achieved through a process-oriented training that includes facts and reflection both on a professional and a personal level [26,37]. A documented, sustainable change occurred among the health professionals at the intervention sites. A shift toward a more facilitating and less regulating attitude occurred up to one year after the intervention [26,27]. This change in the attitudes of caregivers may be the reason why the mothers in the IG perceived stronger professional support from them than did the mothers in the CG, regardless of whether they had a cesarean birth or a normal birth.

Mothers in the IG having had caesarean birth reported stronger for the "Taking in baby" factor than mothers in the CG did three days after birth. This suggests that mothers in the IG were better prepared to meet the difficulties of having had a caesarean birth. The processoriented training may encourage healthcare professionals to be more open to childbirth as a normal life event [48] and to be more aware of the psychosocial process of becoming a mother $[19,20]$ despite mode of delivery. This wider perspective could help healthcare professionals to prepare women for becoming mothers, strengthen their caretaking capacity. It would also support their efforts in supporting mothers to reflect on difficulties before they give birth. Mothers with a caesarean birth has been reported to have low self-esteem [49-51] as well as low self-efficacy concerning breastfeeding and caring for the baby $[49,50]$, and difficulty in her contact with her baby [8]. Result of this study suggest that improved professional support during pregnancy could have a positive influence on mothers with a cesarean birth and decrease the risk of low self-esteem and self-efficacy that might arise in relation to becoming a mother. Through receiving improved professional support the IG mothers seem better prepared to meet the difficulties of a cesarean birth.

Mothers giving birth by operative delivery are more likely to suffer from psychosocial health problems after childbirth [3,5,45], and they seem more self-oriented and less self-confident when caring for the baby two months after birth [50], which may negatively influence mother-infant interaction $[8,17]$. Normal birth may involve a number of physiological adaptations in both the mother and the child $[10,11,52-56]$ that may be delayed after a cesarean birth. Mothers with a cesarean birth will be affected by postoperative pain and pain relief medication, and consequently, they may be less alert and interactive in their encounters with the baby from the start [7,9]. Furthermore, the postnatal behavior of a baby born by cesarean birth may not be as optimal as the postnatal behavior of a baby after a vaginal birth [6] Our result also implies that having a cesarean birth may have a negative impact on the mother's relationship with the baby. Another reason for this negative impact may be because mothers with a cesarean birth have a less mature and pulsatile oxytocin release pattern at breastfeeding compared to mothers with a normal birth [10]. Oxytocin has been suggested to have a positive effect on prenatal bonding [57] and on maternal bonding behaviors [58]. However, mothers in the IG with a caesarean birth reported higher on the "Taking in baby" factor three days after birth. Suggesting that improved professional support from the antenatal midwife and postnatal nurse may strengthen mothers' in relation to and feelings for their baby and buffer the negative effects of caesarean birth in relation to the baby.

Mothers with a normal birth showed somewhat stronger significant differences concerning both perceived professional support and relation to and feelings for the baby some days after birth and at three months after birth. At nine months after birth, this difference was even more striking when comparing perceived professional support. However, mothers with a cesarean birth in the IG showed more significant differences regarding their reported feelings for and their relationship to the baby at nine months after birth than did mothers with a normal birth. This could indicate that despite the small sample size of the caesarean birth group, the intervention had an important impact for these mothers' relation to and feelings for their baby. Considering that mothers with a cesarean birth seems more vulnerable in relation to their baby $[6-8,17,18,50]$, these results might be an important clinical aspect to consider. Using Cohen's guidelines $[41,42]$ the statistically significant results of this study were found to have moderate to small effect size suggesting that our results may have clinical significance.

The larger study was not originally designed to identify differences between women exposed to various modes of giving birth. The results from the present study should be considered as hypothesis generating, and larger trials should be performed. There were also a number of dropouts during the data collection, which is common in longitudinal studies, and may affect the robustness of the results. The three questionnaires used were similar, which could lead to mothers concluding that they had already answered the questionnaire, accounting for the increase in dropouts. Mothers with a caesarean birth are more vulnerable in many aspects and this may be a reason for the lower response rate for this group. However, despite the small sample 
size and the dropouts, there were some differences between the IG and the CG among the mothers with a caesarean birth. This may suggest that the intervention had a clinically important impact, since a smaller sample size raises concerns of not detecting real differences rather than having false significant results [59]. Just a few of our comparative analyses were statistically significant; however, there is a tendency in the result that could suggest a real effect. Our result presents a way for professionals to address the increased vulnerability of a caesarean birth, and is of interest to report. Perceived professional support and maternal feelings for the baby are highly complex issues in research. The development of the scales was based on recommended practices such as underlying theoretical variables [43].In order to gain acceptability and face validity for the items and statements used in this study, a pilot study was performed. We also consulted an expert group of midwives, pediatric nurses, pediatricians, and obstetricians for content validity. Furthermore, the items for professional support correlate well with the mothers' perceptions of professional support [60]. Cronbach's alpha shows a strong internal consistency with the items used. Factor analysis for data reduction was used to decrease the number of statistical tests, and thus, reduce the risk of mass significance and increase the robustness of the results $[38,39]$. The tentative naming of the three factors was completed after careful comparison of our findings with earlier research concerning attachment, bonding, and maternal development $[15,19,40]$. Further research to validate the factorial solutions and to gain an in-depth understanding of the mothers' perceived feelings for and relationship to their babies is ongoing [44]. The statistically significant results of this study were also tested for clinical significance using Cohen's guidelines [41,42], since statistical significance does not automatically mean clinical significance [61]. However, to establish the true clinical importance of our result, further research is needed.

\section{Conclusions}

Result of this study suggests that improved professional support during pregnancy may buffer some negative effects of caesarean birth for first-time mothers in relation to and feelings for the baby. Processoriented training program in professional support in childbearing had a positive influence on how first-time mothers' perceived professional support offered by antenatal midwives and postnatal nurses. The emotional part of professional support seems most important during pregnancy for first-time mothers with a caesarean birth. First-time mothers who were cared for by trained healthcare professionals reported stronger for relation to and feelings for the baby, especially important for mothers having had a caesarean birth.

\section{Competing Interests}

The authors declare that they have no competing interests.

\section{Authors' Contributions}

Stina Thorstensson performed the statistical analysis and drafted the manuscript. Eva Nissen participated in the design of the study and in the completion of the manuscript. Anette Ekstrom participated in the design of the study, data collection, and the completion of the manuscript.

\section{Authors' Information}

Stina Thorstensson is a lecturer at the School of Life Sciences, University of Skovde, and a doctoral student at Örebro University. Her main research interests are social and professional support and the importance and impact that support can have for mother-infant interaction and for childbirth outcomes.
Eva Nissen is an associated professor at the Department of Women's and Children's Health, Division of Reproductive and Perinatal Health Care, Karolinska Institutet, Stockholm, Sweden. Her main research interests are breastfeeding and the influence of different care routines.

Anette Ekström is an associated professor at the School of Life Sciences, University of Skövde, Sweden. Her main research interests are breastfeeding, support during childbirth, and mother-infant interaction.

\section{Acknowledgements}

This study was supported by the Skaraborg Institutet for Research and Development; the School of Life Sciences of the University of Skövde; the Primary Care Unit in Skaraborg and the Science Committee, Central Hospital, Skövde; the Strategic Research Programme in Care Sciences, Karolinska Institutet and the Board of Research for Health and Caring Sciences, Swedish Research Counci [grant nos., K1999-27P-13085-01A and K2001-27P-13085-036]. We wish to thank Professor Ann-Marie Widström for helpful discussions, support, and constructive criticism. We also wish to thank statistician Ann SofiMatthiesen for her valuable contributions to our discussions.

\section{References}

1. The Swedish National Board of Health and Welfare. Medical Birth Register.

2. Wang BS, Zhou LF, Coulter D, Liang H, Zhong Y, et al. (2010) Effects of caesarean section on maternal health in low risk nulliparous women: a prospective matched cohort study in Shanghai, China. BMC Pregnancy Childbirth 10: 78

3. Fisher J, Astbury J, Smith A (1997) Adverse psychological impact of operative obstetric interventions: a prospective longitudinal study. Aust N Z J Psychiatry 31: 728-738.

4. Clement S (2001) Psychological aspects of caesarean section. Best Pract Res Clin Obstet Gynaecol 15: 109-126.

5. Mutryn CS (1993) Psychosocial impact of caesarean section on the family: a literature review. Soc Sci Med 37: 1271-1281.

6. Lobel M, DeLuca RS (2007) Psychosocial sequelae of caesarean delivery: review and analysis of their causes and implications. Soc Sci Med 64: 2272 2284.

7. Tulman LJ (1986) Initial handling of newborn infants by vaginally and cesareandelivered mothers. Nurs Res 35: 296-300.

8. Rowe-Murray HJ, Fisher JR (2001) Operative intervention in delivery is associated with compromised early mother-infant interaction. BJOG 108: 10681075.

9. Karlström A, Engström-Olofsson R, Norbergh KG, Sjöling M, Hildingsson (2007) Post- operative pain after caesarean birth affects breastfeeding and infant care. J Obstet Gynecol Neonatal Nurs 36: 430-440.

10. Nissen E, Uvnäs-Moberg K, Svensson K, Stock S, Widström AM, et al (1996) Different patterns of oxytocin, prolactin but not cortisol release during breastfeeding in women delivered by caesarean section or by the vaginal route. Early Hum Dev 45: 103-118.

11. Nissen E, Gustavsson P, Widström AM, Uvnäs-Moberg K (1998) Oxytocin prolactin, milk production and their relationship with personality traits in women after vaginal delivery in caesarean section. J Psychosom Obstet Gynaecol 19 : 49-58.

12. Raphael-Leff $J$ (2001) Psychological processes of childbearing. (4thedn), The Anna Freud Centre, Guildford.

13. Cassidy J, Shaver PR (1999) Interactional and contextual determinants of attachment security: Handbook of Attachment. Theory, research and clinical application. Guildford Press, New York.

14. Rees C (2008) Children's attachment. Paediatr Child Health 18: 219-226.

15. Klaus MH, Kennell JH, Klaus PH (1995) Bonding: Building the foundations of secure attachment and independence. Addison-Wesley Publications, Massachusetts.

16. Bystrova K, Ivanova V, Edhborg M, Matthiesen AS, Ransjö-Arvidson AB, et al (2010) Early contact versus separation: Effects on mother infant interaction one year later. Birth 36: 97-109. 
Citation: Thorstensson S, Nissen E, Ekstrom A(2012) Professional Support in Pregnancy Influence Maternal Relation to and Feelings for the Baby after Cesarean Birth: An Intervention Study. J Nurs Care 1:112. doi:10.4172/2167-1168.1000112

Page 9 of 9

17. DiMatteo MR, Morton SC, Lepper HS, Damush TM, Carney MF, et al. (1996) Caesarean childbirth and psychosocial outcomes: A meta analysis. Health Psychol 15: 303-314.

18. Garel M, Lelong N, Kaminski M (1987) Psychological consequences of caesarean childbirth in primiparas. J Psychosom Obstet Gynaecol 6:197-209.

19. Mercer RT (2004) Becoming a mother versus maternal role attainment. J Nurs Scholarsh 36: 226-232.

20. Seefat-van Teeffelen A, Nieuwenhuijze M, Korstjens I (2011) Women want proactive psychosocial support from midwives during transition to motherhood: a qualitative study. Midwifery 27: e122-127.

21. Hupcey JE (1998) Clarifying the social theory-research linkage. J Adv Nurs 27 1231-1241.

22. Hertfelt Wahn E, von Post I, Nissen E (2007) A description of Swedish midwives' reflections on their experience of caring for teenage girls during pregnancy and childbirth. Midwifery 23: 269-278.

23. Kahn R, Antonucci T (1980) Convoys of the life course: attachment, roles and social support:Lifespan Development and Behavior. Academic Press, New York.

24. Feeley $N$ (2001) Infant, mother and contextual factors related to mothers interactions with their very-low-birth weight infants. McGill University, Montreal.

25. Oakley A (1994) Giving support in pregnancy; the role of research midwives in a randomized controlled trial: Midwives, Research and Childbirth. (Volume 3), Chapman and Hall, London.

26. Ekström A, Matthiesen AS, Widström AM, Nissen E (2005) Breastfeeding attitudes among counselling health professionals. Scand J Public Health 33: 353-359.

27. Ekström A, Widström AM, Nissen E (2005) Process-oriented Training in Breastfeeding Alters Attitudes to Breastfeeding in Health Professionals. Scand J Public Health 33: 424-431.

28. Ekström A, Kylberg E, Nissen E (2011) A process-oriented breastfeeding training program for health professionals to promote breastfeeding: an intervention study. Breastfeed Med 7: 85-92.

29. Sauls DJ (2002) Effects of labor support on mothers, babies and birth outcomes. J Obstet Gynecol Neonatal Nurs 31: 733-741.

30. Zanna M, Rempel JK (1986) Attitudes, a new look at an old concept: The Socia Psychology of Knowledge. University Press, Cambridge, NY.

31. Ekström A, Widström AM, Nissen E (2006) Does continuity of care by welltrained breast feeding counselors improve a mother's perception of support. Birth 33: 123-130.

32. Ekström A, Nissen E (2006) A mother's feelings for her infant are strengthen by excellent breast feeding counseling and continuity of care. Pediatrics 118 : 309-314.

33. Ekström, A., Guttke, K, Lenz M, Hertfelt-Wahn E (2011) Long term effects of professional breastfeeding support - An intervention. Int J Nurs Midwifery 3 : 109-117.

34. Schulz KF, Altman DG, Moher D, CONSORT Group (2010) CONSORT 2010 statement: updated guidelines for reporting parallel group randomized trials. Obstet Gynecol 115: 1063-1070.

35. Ekström A, Widström AM, Nissen E (2003) Duration of breastfeeding in Swedish primi- parous and multiparous women. J Hum Lact 19: 172-178.

36. Ekström A, Widström AM, Nissen E (2003) Breastfeeding support from partners and grandmothers: perceptions of Swedish women. Birth 30: 261-266.

37. Jerlock M, Falk K, Severinsson E (2003) Academic nursing education guidelines: tool for bridging the gap between theory, research and practice. Nurs Health Sci 5: 219-228.

38. Polit DF, Beck CT (2004) Nursing research: Principles and methods. (7thedn), Lippincott Williams \& Wilkins, Philadelphia

39. Cohen L, Manion L, Morrison K (2007) Research methods in education. (6thedn), Rothledge, Abingdon, UK.
40. George C, Solomon J (1999) Attachment and care giving the care giving behavioral system, In: Cassidy J, Shaver PR (Eds) Handbook of Attachment: Theory, Research and Clinical Applications, Guildford press, New York.

41. Kazis LE, Anderson JJ, Meenan RF (1989) Effect sizes for interpreting changes in health status. Med Care 27: S178-S189.

42. Cohen J (1988) Statistical power analysis for the behavioral sciences. (2ndedn), Lawrence Erlbaum Associates, Inc New Jersey.

43. De Vellis RF (2003) Scale Development: Theory and applications. (2ndedn) Sage Publications Inc, California.

44. Thorstensson S, Hertfelt-Wahn E, Anette E, Langius-Eklof A (2012) Evaluation of the Mother-to-Infant relation and feeling scale: Interviews with firsttime mothers' for feelings and relation to their baby three days after birth International Journal of Nursing and Midwifery 4: 8-15.

45. Coreil J, Bryant CA, Westover BJ, Bailey D (1995) Health professionals and breastfeeding counseling: client and provider views. J Hum Lact 11: 265-271.

46. Wahn EH (2007) Teenage childbearing in Sweden: support from social networks and midwives. Karolinska Institutet, Department of Women's and Children's Health, Division of Reproductive and Perinatal Health Care, Stockholm.

47. Shumaker SA, Brownell A (1984) Towards a theory of social support: closing conceptual gaps. J of Social Issues 40: 11-36.

48. Bryar R, Sinclair M (2011) Theory for midwifery practice. (2ndedn), Palgrave MacHoundsmill, Basingtoke.

49. Aluş Tokat M, Okumuş H, Dennis CL (2010) Translation and psychometric assessment of the breast-feeding Self-Efficacy Scale- Short Form among pregnant and postnatal women in Turkey. Midwifery 26: 101-108.

50. Garel M, Lelong N, Kaminski M (1988) Follow-up study of psychological consequences of caesarean childbirth. Early Hum Dev 16: 271-282.

51. Dennis CL (2006) Identifying predictors of breastfeeding self-efficacy in the immediate postpartum period. Res Nurs Health 29: 256-268.

52. Widström AM, Ransjö-Arvidson AB, Christensson K, Matthiesen AS, Winberg $J$, et al. (1987) Gastric suction in healthy newborn infants. Effects on circulation and developing feeding behaviour. Acta Paediatr Scand 76: 566-572.

53. Matthiesen AS, Ransjö-Arvidson AB, Nissen E, Uvnäs-Moberg K (2001) Postpartum maternal oxytocin release by newborns: effects of hand massage and sucking. Birth 28: 13-19.

54. Jonas W, Nissen E, Ransjö-Arvidson AB, Matthiesen AS, Uvnäs-Moberg $K$ (2008) Influence of oxytocin or epidural analgesia on personality profile in breastfeeding women: a comparative study. Arch Womens Ment Health 11 : 335-345.

55. Jonas K, Johansson LM, Nissen E, Ejdebäck M, Ransjö-Arvidson AB, et al. (2009) Effects of intrapartum oxytocin administration and epidural analgesia on the concentration of plasma oxytocin and prolactin, in response to suckling during the second day postpartum. Breastfeed Med 4: 71-82.

56. Faxelius G, Hägnevik K, Lagercrantz H, Lundell B, Irestedt L (1983) Catecholamine surge and lung function after delivery. Arch Dis Child 58: 262266.

57. Levine A, Zagoory-Sharon O, Feldman R, Weller A (2007) Oxytocin during pregnancy and early postpartum: individual patterns and maternal-fetal attachment. Peptides 28: 1162-1169.

58. Feldman R, Weller A, Zagoory-Sharon O, Levine A (2007) Evidence for neuroendocrinological foundation of human affiliation: plasma oxytocin levels across pregnancy and the postpartum period predict mother-infant bonding Psychol Sci 18: 965-970.

59. Bland M (2000) An introduction to medical statistics. (3rdedn), Oxford University Press, Oxford

60. Bäckström CA, Wahn EIH, Ekström AC (2010) Two sides of breastfeeding support: experiences of women and midwives. International Breastfeed J 5: 20 .

61. Crosby RD, Kolotkin RL, Williams GR (2003) Defining clinically meaningful change in health-related quality of life. J Clin Epidemiol 56: 395-407. 